\title{
Interplay between nutrigenomics and diabetes: a mini review
}

\begin{abstract}
Diabetes Mellitus, regarded as a silent killer, has been demonstrated to be a prime global health concern with a projected rise in prevalence from 171 million in 2010 to 366 million in 2030. Correspondingly, dietary management has shown to be a cornerstone modality in the attainment of good glycemic control in diabetes, and Nutrition/Diet remains a key player in diabetes prevention and management. In this light, of course, evidences from prospective observational studies, clinical trials and experimental findings have reported ameliorating ability of a number foods and nutrient on diabetes. However, a major concern is the genetic variability of individuals and their responses to these functional foods. Therefore, it has become necessary to understand how nutrients act at the molecular level which in turn involves a cascade of nutrient-related interactions at the gene, protein and metabolic levels. Overall, a Nutrigenomic approach provides; a snapshot showing genes that are switched on/off (the genetic potential) at any given moment, and the method to determine the influence of nutrients on gene/protein expression. Nutrigenomic has opened a new future to screen the genetic background, to monitor the transcriptome, proteome and metabolome and to ultimately develop dietary strategies which are targeted to supply the optimum nutrition and therapeutics for single individuals. Therefore, it is vital to support researches in the field of nutrigenomics in response to the prevalence of diabetes mellitus. Hence, the present review provides an understandings vis-à-vis the role of nutrient-gene interactions in diabetes, treatment, prevention and pathogenesis.
\end{abstract}

Keywords: nutrigenomics, diabetes, nutrigenomics and diabetes, nutritional therapy, pancreatic
Volume 7 Issue I - 2020

\author{
Nwawuba Stanley Udogadi,' Mohammed \\ Khadija Abdullahi ${ }^{2}$ \\ 'Department of Biochemistry, Madonna University Okija, \\ Nigeria \\ ${ }^{2}$ Department of Biochemistry, University of Ibadan, Nigeria
}

Correspondence: Nwawuba Stanley Udogadi, Department of Biochemistry, Faculty of Natural Science, Madonna University, Okija, Nigeria, Tel +234-8065699068,

Email nwawubastanle@gmail.com

Received: February 21, 2020 | Published: March 20, 2020
Abbreviations: DM, diabetes mellitus; EGCG, epigallocatechin gallate; T1DM, type $1 \mathrm{DM}$; T2DM, type $2 \mathrm{DM}$; NCD, non-communicable diseases; HGP, human genome project; PDX1, pancreatic and duodenal homeobox 1; GLUT2, glucose transporter 2; SUR1, sulfonylurea receptor-1

\section{Introduction}

Diabetes mellitus (DM) is a metabolic disorder resulting mainly from either the inability of the pancreas to secrete a hormone (insulin) that is responsible for mopping the level of blood sugar, or situation arising from the constellation of insulin insensitivity, resistant and excessive secretion of a hormone called glucagon. ${ }^{1}$ As previously reported, DM remains a world health challenge with an estimated increase in incidence ranging from 171 million in 2010 to 366 million in $2030 .^{2}$ Both the number of cases and its prevalence has been indicated to be on a rapid increase over the years, and as a consequence, DM is considered a silent killer. ${ }^{2,3}$ In an attempt to reduce the menace of diabetes mellitus, several approached has been tested and adopted for the prevention and treatment, including nutritional therapy. However, a key challenge that demands attention is genetic variability. Therefore, an approach which recognizes the interplay between nutritional therapy and genetic factor has become very necessary. This necessity brings nutrigenomics to the forefront Hence, nutrigenomics offers personalized nutritional advice, develops specialized diet/nutrient for a populations or for individuals, and in general, it is regarded as personalized nutrition for the treatment and prevention of several disease conditions including DM. ${ }^{3} \mathrm{~A}$ Nutrigenomic approach finds huge application to the questions of human health and disease, and it is key to understanding the intricacies of interplay between basic metabolic activities and external determinant in disease processes. ${ }^{4}$ It has been demonstrated that, nutrigenomics is critical for the advancement of more targeted strategies in the treatment and prevention of diseases. ${ }^{4}$

Therefore, nutrigenomics research is presently the mainstay owing to the fact that food-derived bioactive components considerably alters genomic, proteomic and metabolic changes. ${ }^{5}$ Nutrigenomics provides an understanding as to how bioactive compounds of a particular diet influences the expression of genes, leading to either activation or suppression. ${ }^{6,7}$ Following the understanding on the interplay between nutrition, genes and diseases provided by nutrigenomics, quite a number evidences exists to this effect. Mediterranean Diet, has been showed to reduce cardiovascular risk factors and stroke prevalence in people with polymorphisms strongly associated with type $2 \mathrm{DM}{ }^{8}$ flavanol, epigallocatechin gallate (EGCG) has been demonstrated to guard insulin secretory cells from pro-inflammatory cytokineinduced toxicity via modulation of $\beta$ cell CLL/lymphoma 2 (BCL-2) expression, ${ }^{9,5}$ and Biotin food sources including Spinach, egg, sweet potato and almonds, enhances insulin secretion and islet function via the gene interaction by signaling an increase in the following gene FOXA2 (Forkhead Box A2), HNF-4 $\alpha$ a nuclear transcription factor, and Calcium Channel Neuroendocrine/Brain-Type, Alpha-1 Subunit (CACNA1D).${ }^{10}$ Therefore, it is vital to support researches in the field of nutrigenomics in response to the prevalence of diabetes mellitus. Hence, the present research provides an understandings vis-à-vis the relationship between nutrition and gene interactions in the treatment, prevention and pathogenesis of diabetes. 


\section{Diabetes}

Diabetes Mellitus (DM) regarded as a serious chronic disease, and its vastly categorized into two (2) etiopathogenetic: Type 1 DM (T1DM) and type 2 DM (T2DM), respectively). ${ }^{1,8}$ T1DM also considered as insulin-dependent diabetes ${ }^{11}$ or juvenile-onset diabetes, it's a consequence of a pancreatic $\beta$ cells dysfunction, and as a result, affected persons depends on exogenous insulin. ${ }^{8}$ One of the key player to development of T1DM, its genetic predisposition via environmental influences in the form of dietary components. ${ }^{12}$ On the other hand, $\mathrm{T} 2 \mathrm{DM}$ is a consequence of situations arising from the constellation of insulin insensitivity, resistant and excessive secretion of a hormone called glucagon. ${ }^{1}$ Its etiology could be as a result of complex combinations of genes, environmental factor (diet) and the interplay between these genes and environmental factors. ${ }^{8} \mathrm{DM}$ regarded as a serious public health challenge, remains among the top four priorities of non-communicable diseases (NCD) targeted for action by world leaders. ${ }^{1}$ Based on existing report, it has been demonstrated that the incidence of diabetes has rapidly been on the rise over the years and as such, it is described to be a silent killer disease affecting large population of people around the world. ${ }^{12}$ The rise in worldwide prevalence is reported to grow from 171 million in $2010,{ }^{13}$ to 382 million in $2013,{ }^{14}$ and it is expected to rise to 592 million by $2035 .{ }^{1,14}$ Clearly, DM poses as huge global health challenge that has assumed pandemic proportion affecting the developing countries more than the developed countries. ${ }^{1,12,13}$ Owing to this in part, the important role of diet/nutrient in diabetes management and prevention was recognized by the medical society particularly the American Diabetes Association and new recommendations were then focused on individualized eating plans targeted at reaching specific biomarkers. ${ }^{15}$

\section{Nutrigenomic}

Environmental factor including diet/nutrition is recognized to be a key contributor to human health and several disease conditions. ${ }^{16}$ As such, nutritional research has continued to elucidate and provide an understanding of how to maintain a good health, and effectively ameliorate several disease conditions using dietary approach. The completion of the Human Genome Project (HGP) led to advancement in nutritional approach, and the opening of frontier on targeted nutritional therapy. According to Sales et al., ${ }^{6}$ the conclusion of HGP led to new insights on the role of diet/nutrition on human health, and as a result the following postulations were made; ${ }^{6}$

i. Gene expression in response to metabolic process, at cellular level, could have the ability to influence individual health

ii. Could gene expression and metabolic response be as a consequence of the interaction between genotype and environment/nutrient?

iii. Could the understanding of the interaction between diet/nutrition and gene results to recommendation of specific diets for a particular person? Nutrigenomics provides the exact response the postulations and questions raised.

Nutrigenomics, sometimes called nutritional genomics is an emerging area of research, and in recent years, the field has received enormous attention. ${ }^{4}$ It focuses on the investigation on the area of nutrition that adopts molecular approaches to elucidate responses from certain diet exposed to either individual or population groups. ${ }^{17,18}$ A number of definitions on nutrigenomics exist, and the all tends towards same meaning; influence of nutrition/nutrient on an individual genome hence, nutrigenomics. Accordingly, Barnett \&
Ferguson $^{3}$ nutrigenomics is considered as the interactions between diet/nutrition and an individual's genome, and the downstream effects on his or her phenotype. ${ }^{3}$ Nutrigenomics offers personalized nutritional advice, develops specialized diet/nutrient for a populations or for individuals, and in general, it is regarded as personalized nutrition for the treatment and prevention of several disease conditions including DM. ${ }^{3}$ Nutrigenomics is critically involved with developing scientific framework to enhance a better understanding on genetic contributions to such preferences, requirements, and responses to diet/nutrition, and also advance future methods for consumers to assess their health and nutritional status. ${ }^{19,20}$ Correspondingly, it recognizes that a particular dietary choice for one person may be unsuitable for the other, ${ }^{3}$ and similar to its analogous, pharmacogenomics, nutrigenomics has been demonstrated to possess the ability of identifying genetic predictors that responds to diets in relation to disease conditions and its applicability in the milieu of personalized nutrition have popular appeal. $^{4}$

\section{Interplay between of nutrient/gene interactions (nutrigenomics) vis-à-vis diabetes mellitus}

Several studies have shown and established the fact that diet/ nutrition plays a prominent role in the etiology and prevalence of disease conditions including diabetes through an interaction of multiple genes in response to the diet. ${ }^{2,8}$ Therefore, the identification and analysis of the interaction between diet/nutrient and genes (nutrigenomics) are important steps toward the understanding of DM etiopathogenesis, prevention and management. ${ }^{8,3}$ In establishing the interplay between diet and gene interactions, nutrigenomics greatly relies on high-throughput technologies and methodologies in genomics, transcriptomics, proteomics and metabolomics for the analysis of targeted genes and metabolites that responds to the diet/ nutrient, and also the analysis of bioactive compounds present in foods as well as how they affects the human metabolism, nutritional homeostasis and molecular events involved in nutrition-related diseases, such as diabetes. ${ }^{8}$ In molecular nutrition perspective, nutrients could be regarded as signaling molecule capable of either transmitting or translating dietary signals into changes in the gene expression as well as synthesis of proteins and metabolites via appropriate cellular sensing mechanisms. ${ }^{5}$ Generally, diet/nutrients has the ability to affect gene expression via the following mechanism as stated by Genoveva et al., ${ }^{8}$ : (i) directly (ii) through their metabolites and (iii) through signal transduction molecules. This is depicted in figure 1, as the figure shows, food/diet/nutrient influences gene expression leading to prevention and treatment of diabetes (glucose hemostasis). The understanding of the interplay between diet/ nutrient and human genome has helped establish precision nutritional therapeutic approaches to the study of health and disease. ${ }^{21}$ Clearly, the potentials provided by evidence-based personalized or precision nutritional therapeutic approaches towards the maintenance of good health as well as the amelioration of several disease states including diabetes, continues to be an important healthcare goal. ${ }^{3}$ Evidence has shown that several dietary-bioactive compounds possesses the ability to regulate gene expression directly or indirectly through induction of metabolites or signaling molecules that influence complex metabolic pathways involved in the development of diabetes. ${ }^{5}$

In addition, quite a number of studies using humans, animals, and cell cultures model, have revealed the interplay between diet/nutrient and gene expression involved in diabetes. Naringin, present in large amount in citrus fruits, and some vegetables, has been demonstrated to reduce insulin resistance, improve $\beta$ cell function, and acts as 
potent a hypoglycemic candidate via gene interaction by improving the peroxisome proliferator-activated receptors (PPPAR- $\gamma$ ), ${ }^{22}$ Biotin food sources including spinach, egg, sweet potato and almonds, enhances insulin secretion and islet function via the gene interaction by signaling an increase in the following gene FOXA2 (Forkhead Box A2), HNF-4 $\alpha$ a nuclear transcription factor, and Calcium Channel Neuroendocrine/Brain-Type, Alpha-1 Subunit (CACNA1D), ${ }^{10}$ Taurine from animal foods, such as meat, fish and dairy as the main sources, increases Insulin secretion and insulin synthesis via gene interaction by signaling an increase in the following gene PDX1 (pancreatic and duodenal homeobox 1), Glucose transporter 2 (GLUT2), and sulfonylurea receptor-1 (SUR1) encoded by the ABCC 8 gene, ${ }^{23}$
Dietary amino acids modulates the insulin resistant and type 2 diabetes mellitus by influencing gene and protein expression in pancreatic $\beta$ cell of islets of langerhans, ${ }^{24}$ Vitamin $\mathrm{D}$ promotes insulin secretion and cell protection by attenuating the expression and activity levels of IL1 , IL-6 and TNF- $\alpha$ in T2DM, ${ }^{5}$ naturally occurring bioactive chemicals including flavonoids, carotenoids, coumarins, and phytosterols; and zoochemicals such as eicosapentaenoic acid and docosahexaenoic acid) regulate gene expression in diverse ways, ${ }^{21}$ and Sorghum improves insulin sensitivity via (PPAR- $\gamma$ ) from adipose tissue, downregulates of TNF- $\alpha$, up-regulates adiponectin, and improves lipid profile status. ${ }^{25,2}$

\section{Food and Diet}

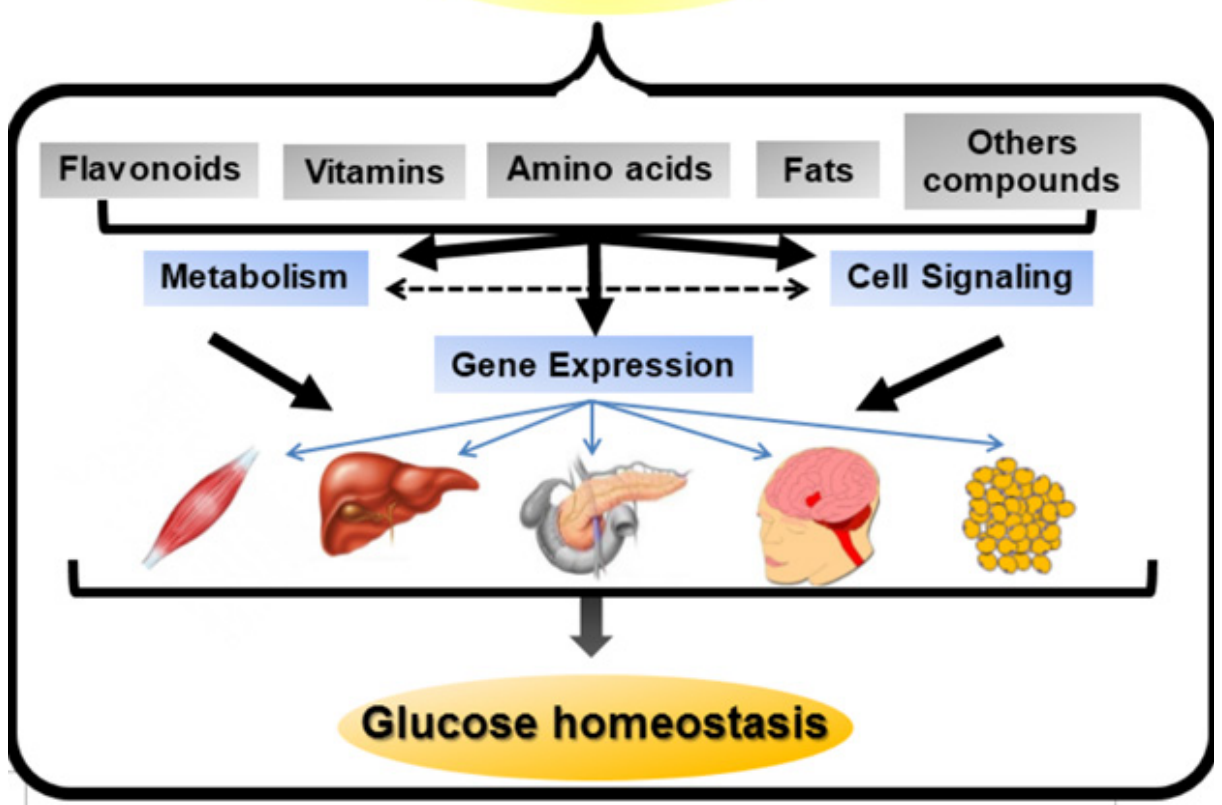

Figure I Diet-Gene expression mechanisms. ${ }^{8}$

\section{Conclusion}

The knowledge of how food interferes with the genetic code and how the organism responds to these interferences is what nutrigenomic brings. Therefore, it is imperative to improve research in the field of nutrigenomics because these discoveries will deliver a framework for the development of genotype-dependent food health promotion strategies/evidence-based personalized or precision medicine through dietary intervention, and the design of dietetic approaches for the prevention and management of diabetes mellitus.

\section{Acknowledgments}

None.

\section{Conflicts of interest}

The authors declared that there are no conflicts of interest.

\section{Funding}

None.

\section{References}

1. Nwozo SO, Ikpeme GE, Nwawuba SU. Functional dietary supplementation of Okara (soybeans residue) on streptozotocin induced diabetes mellitus in male wistar rats. Global Journal of Medical Research: F Diseases. 2019;19(5):17-28.

2. Nwawuba SU, Nwozo SO, Mohammed KA. Dietary management of diabetes mellitus with focus on Nigeria. International Journal of Diabetes Research. 2019;2(1):26-32.

3. Barnett MPG, Ferguson LR. Nutrigenomics: integrating genomic approaches into nutrition research. Molecular Diagnostics. 2017:305326.

4. Ferguson JF, Allayee H, Gerszten RE, et al. Nutrigenomics, the microbiome, and gene-environment interactions: new directions in cardiovascular disease research, prevention, and treatment. a scientific statement from the American heart association. Circ Cardiovasc Genet. 2016;9(3):291-313.

5. Ravindra PV, Jaiswal N, Janhavi P. Nutrigenomics: diabetes perspective. Asian Journal of Emerging Research. 2019;1(1):8-11. 
6. Sales NMR, Pelegrini PB, Goersch MC. Nutrigenomics: definitions and advances of this new science. Journal of Nutrition and Metabolism. 2014;202759:1-6.

7. Dauncey MJ. Recent advances in nutrition, genes and brain health Proceedings of the Nutrition Society. 2012;71(4):581-591.

8. Berná G, Oliveras-López MJ, Jurado-Ruíz E, et al. Nutrigenetics and nutrigenomics insights into diabetes etiopathogenesis. Nutrients. 2014;6(11):5338-5369

9. Zhang Z, Ding Y, Dai X, et al. Epigallocatechin-3-gallate protects pro-inflammatory cytokine induced injuries in insulin-producing cells through the mitochondrial pathway. Eur J Pharmacol. 2011;670(1):311316.

10. Lazo ML, Larrieta E, German MS, et al. Effects of biotin supplementation in the diet on insulin secretion, islet gene expression, glucose homeostasis and beta-cell proportion. J Nutr Biochem. 2013;24(1):169-177.

11. Dib SA, Gomes MB. Etiopathogenesis of type 1 diabetes mellitus: prognostic factors for the evolution of residual beta cell function. Diabetol Metab Syndr. 2009;1(25):1-8.

12. Nwozo SO, Nwawuba SU. Evaluation of antidiabetic role of bridelia ferruginea methanol leaf extract in streptozocin induced diabetic male wistar rats. Pharmacy \& Pharmacology International Journal. 2019;7(6):264-269.

13. Nwozo SO, Modeme TE, Nwawuba SU. Evaluation of momordica charantia, boerhaavia diffusa and cotreatment on streptozotocin induced diabetes in male wistar rats. International Journal of Biological Sciences. 2018;14(2):66-73.

14. Nwawuba SU, Monago CC, Mejulu KC. Ameliorative effect of aqueous seed extract of delonix regia on hyperglycemia, liver function and lipid profile levels in streptozotocin induced diabetic male wistar rats. Pharmacy \& Pharmacology International Journal. 2019;7(3):126-131.

15. Ordovas JM, Berciano S, Mico V, et al. Diet-Gene interactions in the development of diabetes. Molecular Nutrition and Diabetes. 2016:4150 .
16. Ronteltap A, van Trijp JC, Renes RJ. Consumer acceptance of nutrigenomics-based personalised nutrition. Br J Nutr. 2009;101(1):132144.

17. Pavlidis C, Patrinos GP, Katsila T. Nutrigenomics: a controversy. Appl Transl Genom. 2015;4:50-53.

18. Sharma P, Dwivedi S. Nutrigenomics and nutrigenetics: new insight in disease prevention and cure. Indian J Clin Biochem. 2017;32(4):371373.

19. Kussmann M, Daniel H. Editorial overview. Curr Opin Biotechnol. 2008;19(2):63-65.

20. Kussmann M, Van Bladeren PJ. The extended nutrigenomics understanding the interplay between the genomes of food, gut microbes, and human host. Frontiers in Genetics. 2011;2(21):1-13

21. Mead MN. Nutrigenomics: the genome-food interface. Environ Health Perspect. 2007;115(12):A582-A589.

22. Sharma AK, Bharti S, Ojha S, et al. Up-regulation of PPAR $\gamma$, heat shock protein- 27 and -72 by naringin attenuates insulin resistance, beta-cell dysfunction, hepatic steatosis and kidney damage in a rat model of type 2 diabetes. Br J Nutr. 2011;106(11):1713-1723.

23. Carneiro EM, Latorraca MQ, Araujo E, et al. Taurine supplementation modulates glucose homeostasis and islet function. J. Nutr Biochem. 2009;20(7):503-511

24. Menni C, Fauman E, Erte I, et al. Biomarkers for type2 diabetes and impaired fasting glucose using a nontargeted metabolomics approach. Diabetes. 2013;62(12):4270-4276.

25. Park JH, Lee SH, Chung IM, et al. Sorghum extract exerts an antidiabetic effect by improving insulin sensitivity via PPAR- $\gamma$ in mice fed a high-fat diet. Nutr Res Pract. 2012;6(4):322-327. 